\title{
Significados de madres y padres mexicanos del mismo género en torno a la crianza
} Meanings of Mexican same-sex parents around parenting

(c) $\frac{10(2)(2)}{\mathrm{BY} N \mathrm{NA}}$

Fabiola Rodriguez-Sánchez Jessica Karime Malagon Arias

Fernando Salinas-Quiroz

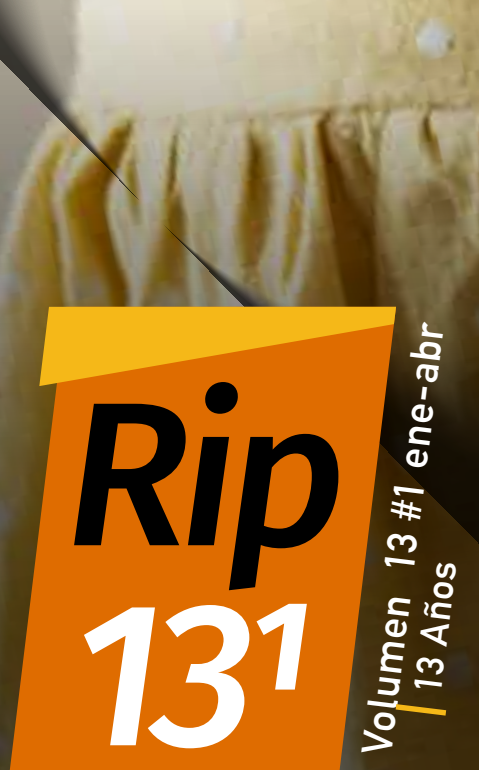

Revista Iberoamericana de

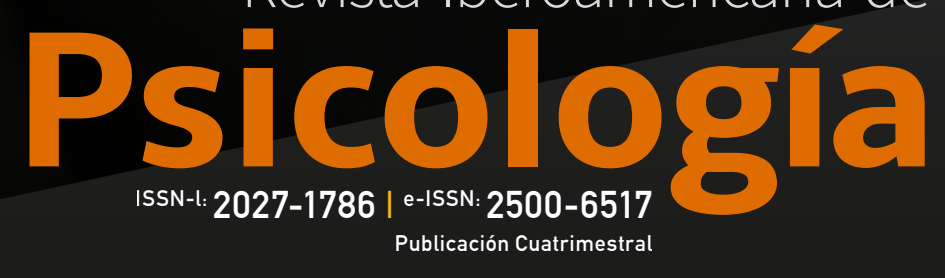


Revista Iberoamericana de

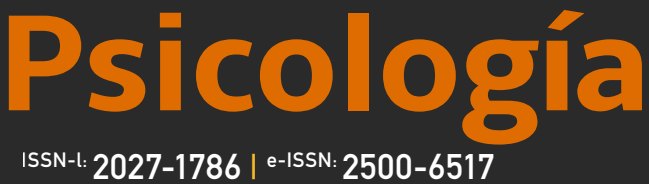
Publicación Cuatrimestral

ID:

10.33881/2027-1786.RIP.13104

Title: Meanings of Mexican same-sex parents around parenting

Título: Significados de madres y padres mexicanos del mismo género en torno a la crianza

Alt Title / Título alternativo:

[en]: Meanings of Mexican same-sex parents around parenting

[es]: $\quad$ Significados de madres y padres mexicanos del mismo género en torno a la crianza

Author (s) / Autor (es):

Rodríguez-Sánchez, Malagon Arias \& SalinasQuiroz

Keywords / Palabras Clave:

[en]: Same-gender parenting; upbringing; early childhood; meanings

[es]: Homoparentalidad; crianza; primera infancia; significados

Submited:

2019-07-09

Acepted:

2020-03-22

\section{Resumen}

Este estudio muestra los significados expresados por tres parejas del mismo género (cuatro mujeres y dos hombres) sobre la crianza de sus hijas e hijos,

quienes tenían entre uno y tres años de edad.

Desde un enfoque hermenéutico-interpretativo, analizamos entrevistas narrativas, resultando en las siguientes categorías: Importancia de verbalizar y explicar; Reglas, límites y consecuencias; Enseñanza y aprendizaje de valores y habilidades sociales; Mantener una relación cercana; Rasgos/ atributos personales valorados (en las y los niños).

Encontramos diversas funciones asumidas en

su labor como educadores, que se vincularon de forma dinámica con lo que esperaban y valoraban en cuanto a aprendizaje y desarrollo. Fue importante para ellos/as complacer, dar gusto y satisfacer las necesidades de sus niñas y niños, sin que esto significara descuidar una alimentación saludable, establecer y mantener reglas y rutinas, así como enseñar habilidades acordes a su edad. Un significado compartido giró en torno al lenguaje y comunicación, pues para los adultos era importante explicar: desde rutinas, hasta verbalizar sentimientos, o hacer explícito su tipo de

familia (tener dos padres o dos madres); siempre considerando lo que puede comprenderse a esa edad. También fue importante para los adultos que sus hijos e hijas fueran alegres y sintieran su amor, manteniendo una relación cercana: cálida, lúdica y afectuosa. Concluimos que estos padres gais y madres lesbianas significaron a la crianza como una tarea amorosa, ardua, permanente

y dirigida a formar sujetos con derechos, con quienes se podía negociar y llegar a acuerdos, aun siendo niños y niñas

\section{Abstract}

This study shows the meanings expressed by three Mexican same-gender couples (four women and two men) about the upbringing of their daughters and sons, who were aged between one and

three years. From a hermeneutic-interpretative approach, we analysed narrative interviews, resulting in the following categories: Importance

of verbalizing and expressing; Rules, limits and consequences; Teaching and learning values and social skills; Maintain a warm relationship; Personal traits/attributes valued (in children). We found that fathers and mothers played different roles in their work as educators, which were linked dynamically with what they expect and value in terms of learning and development. It was important for them to please, to indulge and to satisfy their children's needs, without this neglecting a healthy diet, establishing and maintaining rules and routines, as well as teaching values and skills according to their age. A shared meaning revolved around language and communication: for adults it was important explaining routines, verbalizing feelings, or making explicit their type of family (having two fathers or two mothers); always considering what can be understood at that age. It was also important for our participants that their sons and daughters felt happy and loved through a close, warm, playful and affectionate relationship. We conclude that these gay parents and lesbian mothers meant the upbringing as a loving, arduous and permanent task, committed to forming subjects with rights, with whom they could negotiate and reach agreements

\section{Citar como:}

Rodríguez-Sánchez, F., Malagon Arias, J. K. \& Salinas-Quiroz, F. (2020). Significados de madres y padres mexicanos del mismo género en torno a la crianza. Revista Iberoamericana de Psicología 13(1), 33-44. Obtenido de: https://reviberopsicologia.ibero.edu.co/article/view/1653

Jessica Karime Malagon Arias, [Psi]

BIO:

Pasante de la carrera de Psicología Educativa.

City | Ciudad:

Ciudad de México [mx]
Doctora en Psicología Profesora de tiempo completo.

City | Ciudad:

Ciudad de México [mx]
Fernando Salinas-Quiroz, Pdoc Ps

ORCID:

https://orcid.org/0000-0002-1257-6379

Source | Filiacion:

Universidad Pedagógica Nacional

$\mathrm{BIO}$ :

Investigador Nacional, Sistema Nacional de Investigadores. Doctor en Psicología. Profesor Titular. Fundador y Tesorero de la International Academy for LGBT Psychology and Related Fields. Miembro del Comité directivo del Sexual Orientation and Gender Identity/ Expression Caucus de la Society for Research in Child Development.

City | Ciudad:

Ciudad de México [mx 


\section{Significados de madres y padres mexicanos del mismo género en torno a la crianza}

Meanings of Mexican same-sex parents around parenting

Fabiola Rodríguez-Sánchez Jessica Karime Malagon Arias

Fernando Salinas-Quiroz

\section{Marco teórico}

\section{El papel de los adultos en la crianza}

La Real Academia Española (2018) establece más de diez significados al término criar, definiéndolo como nutrir, cuidar, instruir y educar. La crianza como actividad cultural compleja, debiera asumirse por toda una comunidad o red de apoyo, pues en los primeros años se asientan las bases para lo que nos hace humanos: el lenguaje y nuestra capacidad de vincularnos socioafectivamente. Fue Vygotsky (1934/1995) el primer psicólogo en dar una explicación sólida acerca de la génesis social de las funciones psicológicas superiores, así como del papel del lenguaje y las interacciones sociales en el desarrollo humano. Su legado puede verse en nociones ya clásicas como la de participación guiada de Rogoff (1993), hasta en desarrollos teóricos más recientes como la teoría del self dialógico (Roncancio-Moreno, BermúdezJaimes, \& Uchoa, 2019).

Teóricos del siglo pasado como Bowlby (1996), y Ainsworth, Blehar, Waters y Wall (1978), con su Teoría del Apego realizaron una invaluable aportación sobre la importancia de la figura materna en el desarrollo socioafectivo infantil. Ellos han sido la base de posteriores investigaciones, las que utilizando la observación de interacciones adulto-niño, intentan dar cuenta del fenómeno de la base segura (Duarte, García, Rodríguez, \& BermúdezJaimes, 2016; Salinas-Quiroz \& Costa, 2018). 
Otra mirada desde donde se sigue investigando el papel de los adultos, se encuentra en los estilos parentales o pautas de crianza (Jorge \& González, 2017; Pulido, Castro-Osorio, Peña, \& Ariza-Ramírez, 2013), relacionando dichas formas con el desarrollo socioemocional (Cuervo, 2010) o habilidades sociales (Isaza \& Henao, 2010). Más enfocado en los procesos y contenidos del pensamiento adulto, está el trabajo sobre creencias parentales y teorías implícitas. Su carácter episódico-semántico y biográfico implica asumir que dichos conocimientos se construyen a través de actividades y formatos de interacción social (Rodrigo, 2003). "Los padres no eligen al azar sus prácticas de intervención entre todo un conjunto de posibilidades educativas, sino que su selección refleja un modo especial de entender al niño y sus necesidades" (Triana, 2003). En esta línea, se han documentado diversas ideas sobre el desarrollo, aprendizaje, valores y actitudes; así como sobre las relaciones entre concepciones sobre la crianza con atribuciones, autoeficacia y auto-percepciones como padres/madres (Infante \& Martínez, 2016; Merrifield, Gamble, \& Jin, 2015).

Otras investigaciones de corte cuantitativo se inscriben dentro de las etnoteorías, donde convergen estudios transculturales que analizan diferentes pautas, metas, concepciones y prácticas de crianza (Harkness, y otros, 2011; Da Rocha, Roethle, \& Mochizuki, 2012; Mone, Benga, \& Susa, 2014). Desde una interpretación socialmente situada y etnográfica, diversos estudios recuperan el papel de los contextos en el contenido de las creencias, así como en las prácticas de crianza (Goncalvez \& Franco, 2011; Morgante \& Remorini, 2013). Estas investigaciones otorgan miradas más contextuales a cómo los contenidos del pensamiento adulto van configurándose de forma situada, pero existe poca literatura en este sentido sobre familias con padres/madres del mismo género.

\section{Familias homoparentales}

Diversas investigaciones se han centrado en mostrar cuál podría ser el impacto diferencial de las prácticas de crianza entre padres/madres heterosexuales y homosexuales en el desarrollo de sus hijos/as, a través del uso de estudios longitudinales. Distintos meta-análisis concluyen que no hay evidencia contundente que señale diferencias significativas en cuanto a competencias parentales, o niveles de desarrollo sociocognitivo y emocional (Carneiro, Tasker, Salinas-Quiroz, Leal, \& Costa, 2017; Fedewa, Black, \& Ahn, 2015; Salinas-Quiroz \& Costa, 2018). Incluso se han reportado diferencias a favor de las familias homoparentales en cuanto a mayor igualdad en la distribución de tareas domésticas y de crianza entre la pareja (González, Diez, López, Martínez, \& Morgado, 2013).

Los estudios comparativos han contribuido en la desmitificación y disminución de prejuicios hacia las familias homoparentales; sin embargo, aún son vistas como transgresoras de los mandatos de una sociedad heteronormada. Nosotros reivindicamos la necesidad de comprender lo que ocurre al interior de las familias homoparentales, desde su propia experiencia. En este sentido, González y López (2009) entrevistaron a madres lesbianas y padres gais de hijos/hijas entre $\mathbf{3}$ y 16 años de edad, donde el 64\% eran producto de una unión heterosexual previa y la mitad de las familias era monoparental. Encontraron que sus rutinas eran estables y acordes a su edad; la comunicación, el afecto, el contacto frecuente y cálido con la familia extensa, fueron dimensiones importantes para la vida cotidiana desde la perspectiva de las 28 familias.
En México hay poca investigación sobre familias homoparentales, lo cual se debe en parte a que su visibilización ha implicado un lento proceso. Fue hace diez años que el matrimonio igualitario se legalizó y sólo un año después se permitió adoptar (Salinas-Quiroz \& Costa, 2018) No obstante, a la fecha esto no se ha logrado en todo el país; sin mencionar que en estados como Quintana Roo, Nayarit o Jalisco, únicamente el matrimonio igualitario es legal, pero no se reconoce el derecho a la adopción. Si a ello le agregamos que no es fácil acceder a testimonios de familias homoparentales (Giraldo, 2015), podemos comprender por qué muchos estudios realizados en México son de corte cualitativo con pocos casos.

Haces (2015) reportó que con independencia del vínculo parental (biológico o no), en las entrevistas realizadas a nueve parejas, resaltaba un discurso de afiliación/relación. Para los adultos era importante que dicha relación también fuera visibilizada desde el registro para que los vástagos lleven el apellido de los dos padres/madres. Por su parte, Laguna (2015) profundiza en las ideas de ocho padres gay sobre paternidad y familia, encontrando un significado común relativo al temor y preocupación de que sus hijos/as sufrieran en algún momento de su vida manifestaciones de homofobia; lo cual intentaban contrarrestar con una educación (tanto escolar como familiar) centrada en el respeto a la diversidad de género.

Contreras y Pons (2015) exploraron las experiencias de distintas parejas (heterosexuales, homosexuales y transexuales), encontrando en ellas la necesidad de resignificar múltiples ideas en cuanto a la crianza para construir una concepción más incluyente y respetuosa de las diferencias. Algo similar reportan Salinas-Quiroz et al. (2018), quienes, desde la Teoría del Apego, buscaron comprender por qué padres gais y madres lesbianas obtuvieron altos niveles de sensibilidad y fueron base de seguridad para sus hijos/as.

En síntesis, las investigaciones referidas se han realizado prioritariamente con familias monoparentales (sobre todo mujeres lesbianas), o bien, con parejas con hijos/as mayores a los tres años de edad. En México se requiere aún de mucha investigación, considerando en particular, las dinámicas cotidianas y el mundo simbólico de parejas del mismo género que asumieron vivir de forma conjunta la crianza.

Así, el objetivo de esta investigación es: analizar los significados de padres gais y madres lesbianas sobre la crianza de sus hijos/a entre uno y tres años de edad. Definimos "significados" como construcciones intersubjetivas, culturales y contextuales que se generan, negocian y modifican en el curso de las actividades y relaciones sociales y que se objetivan en narraciones (Arcila, Mendoza, Cañón, \& Jaramillo, 2010).

\section{Método}

\section{Población y muestra}

Se trata de una muestra no probabilística por conveniencia (Otzen \& Manterola, 2017) de cuatro madres lesbianas y dos padres gais con edad promedio de 36 años; nivel socioeconómico medio-alto, escolaridad universitaria y todos residentes en la Ciudad de México. En la Tabla 1 se sintetizan algunas características relevantes de las familias. 
Tabla 1 Características de las/os participantes

\begin{tabular}{|c|c|c|c|c|}
\hline Madre/padre & Edad & Ocupación & Tiempo de pareja & Hijos/as, edad \\
\hline $\begin{array}{l}\text { Lilia } \\
\text { Flor* }\end{array}$ & $\begin{array}{l}47 \\
41\end{array}$ & $\begin{array}{l}\text { Maestra de yoga } \\
\text { Editora de cine }\end{array}$ & Seis años & Julia 3 años \\
\hline $\begin{array}{l}\text { Gabriela* } \\
\text { Eugenia }\end{array}$ & $\begin{array}{l}37 \\
30\end{array}$ & $\begin{array}{c}\text { Free lance } \\
\text { Comercialización de productos }\end{array}$ & Diez años & $\begin{array}{c}\text { Gabriely Paulo (gemelos } \\
19 \text { meses) }\end{array}$ \\
\hline $\begin{array}{l}\text { Noé }^{* *} \\
\text { Gerardo** }\end{array}$ & $\begin{array}{l}30 \\
35\end{array}$ & $\begin{array}{c}\text { Representante Médico } \\
\text { Médico }\end{array}$ & Dos años & Karina 27 meses \\
\hline & & Vía de acceso: *Insemina ción a & tificial ${ }^{*}$ Adopción & \\
\hline
\end{tabular}

\section{Diseño}

Asumir un enfoque hermenéutico-interpretativo implica aspirar a comprender la experiencia de los hechos sociales "objetivados"; cómo las personas significamos el mundo social (Tarrés, 2001). El énfasis se encuentra en el análisis de los significados que los agentes (Denzin \& Lincoln, 2012), en este caso madres y padres homosexuales, construyen en torno a algún aspecto de su vida social, en concreto sobre la crianza. Para develar tales sentidos, se consideran las experiencias cotidianas de las personas, interpretadas y narradas desde su propia perspectiva, enfatizando el contenido de su discurso (Bruner J. , 1990).

\section{Instrumentos de recolección de la información}

Utilizamos la entrevista narrativa ya que permite explorar "el mundo experiencial" de las personas, asumiendo que tales experiencias se estructuran y recuperan en forma narrativo-episódica y semántica (Flick, 2004). La entrevista incluyó preguntas reconstructivo-biográficas (como narrar el proceso de embarazo/adopción); enfocadas en situaciones y rutinas cotidianas (por ejemplo, "¿qué es lo que hacen los fines de semana?"); así como preguntas que requieren de mayor reflexividad (“qqué significa para usted una familia?”).

Este último tipo de preguntas exige una valoración atribucional que se organiza de forma diferente que las otras preguntas pese a "... basarse en las mismas entidades representacionales de base" (Rodrigo, 2003). Como afirma Flick (2004), "mientras que el conocimiento episódico se asocia a situaciones y circunstancias concretas, el conocimiento semántico se basa en supuestos y relaciones que se abstraen a partir de ellas y se generalizan" (p.118).

\section{Procedimiento y Consideraciones éticas}

Tomando en cuenta las dificultades para acceder a familias homoparentales, aprovechamos la orientación homosexual del autor de correspondencia para obtener el apoyo de una organización no gubernamental, denominada "Familias diversas"; asociación civil donde Lesbianas, Gais, Bisexuales y Transexuales (LGBT) conviven y dialogan sobre sus experiencias como padres y madres. Los criterios de inclusión fueron, además de la orientación sexo-afectiva homosexual, que tuvieran hijos/hijas de tres años o menos. Las entrevistas se realizaron individualmente con una duración promedio de dos horas.

\section{Análisis de la información}

Asumimos un análisis de tipo hermenéutico-interpretativo buscando llegar a una comprensión de las ideas en los propios términos de los/ as participantes, sin categorías preestablecidas pero en un proceso continuo de espiral inductivo-deductivo (Weiss, 2007). Desde las primeras lecturas de las trascripciones, nos implicamos al menos dos investigadores, identificando expresiones emblemáticas según nuestras primeras intuiciones. Gracias a la minuciosa y sistemática codificación (Braun \& Clarke, 2006), logramos un conocimiento compartido y fino de nuestros datos, lo cual nos condujo a que arribáramos a interpretaciones más pertinentes. Fuimos seleccionando frases y párrafos que se relacionaran con los conceptos que teníamos en mente (crianza), pero que también resultaban representativos del texto (entrevista) como un todo. Esto nos permitió relacionar los textos entre sí e ir construyendo, descartando y afinando temáticas que pudieran dar cuenta de la diversidad de significados. Las categorías finales enfatizan el contenido del discurso, a partir de analizar la singularidad de los casos.

Discutir los resultados entre colegas heterosexuales y homosexuales, con y sin hijos, enriqueció la interpretación en un ejercicio permanente de explicitar y poner en tela de juicio nuestros propios significados y creencias sobre la crianza. Ello no impidió que lográramos, desde la codificación, niveles adecuados de "dependencia" o "auditability”, en términos de Guba y Lincoln, o de Miles y Huberman respectivamente como se citó en (Sandín, 2003). Es decir, todo el proceso de análisis, desde la codificación hasta la definición de categorías se sometió a una triangulación de investigadores. Explicitar nuestros juicios y decisiones como elemento intrínseco en la investigación cualitativa (Maxwell, 1996), implica mantener un nivel de reflexividad a lo largo del proceso investigativo, lo cual es también una estrategia de "control de calidad” que otorga mayor credibilidad a los resultados (Berger, 2013).

\section{Resultados}

Este apartado está organizado por categorías, cuya definición puede verse en la Tabla 2. A lo largo de la interpretación, incluimos diversos ejemplos y fragmentos de las entrevistas a modo de narración, para ser congruentes con las premisas de una investigación de corte interpretativo. 


\section{Significados de madres y padres mexicanos del mismo género en torno a la crianza}

Tabla 2 Definición de Categorías de análisis

\begin{tabular}{|c|c|}
\hline CATEGORIASS & DEFINICIÓN \\
\hline $\begin{array}{l}\text { Importancia de verbalizary } \\
\text { explicar }\end{array}$ & $\begin{array}{l}\text { Se refiere al papel otorgado al lenguaje como medio de comunicación entre } \\
\text { padres/madres e hijos/as, pero también como forma de anticipación (de rutinas), de } \\
\text { instrucción (explicación de cómo se hace algo) y de objetivación de la realidad (por } \\
\text { ejemplo, nombrar emociones). }\end{array}$ \\
\hline Reglas, límites y consecuencias & $\begin{array}{l}\text { Integra descripciones que muestran la relevancia que tiene para los adultos } \\
\text { implementar estos tres aspectos, así como a la forma en que lo llevan a cabo para que } \\
\text { sus hijas e hijos puedan comprender lo que está permitido en su contexto. }\end{array}$ \\
\hline $\begin{array}{l}\text { Enseñanza y aprendizaje de } \\
\text { valores y habilidades sociales }\end{array}$ & $\begin{array}{l}\text { Incluye expresiones que reflejan el interés que tienen los padres y las madres para que } \\
\text { sus hijos e hijas aprendan ciertos elementos valorados en sus contextos de socialización, } \\
\text { tales como el respeto, la responsabilidad, compartir, esperary tolerar la frustración, } \\
\text { entre otros. }\end{array}$ \\
\hline Mantener una relación cercana & $\begin{array}{l}\text { Se refiere a la importancia que tiene para los adultos hacer sentir bien a sus hijos e hijas } \\
\text { y cubrir sus necesidades físicas y emocionales. Incluye descripciones sobre distintas } \\
\text { formas de demostrarles a fecto, complacerles y darles gusto, así como de reconocer y } \\
\text { valorar sus a vances y aciertos. }\end{array}$ \\
\hline $\begin{array}{l}\text { Rasgos/atributos personales } \\
\text { valorados }\end{array}$ & $\begin{array}{l}\text { Agrupa afirmaciones sobre las cualidades o características personales que los } \\
\text { padres/madres esperan que sus hijos e hijas posean, obien, que ya son identificadas } \\
\text { como parte de su personalidad. Incluye descripciones y atribuciones sobre las } \\
\text { características que consideran como femeninas o masculinas. }\end{array}$ \\
\hline
\end{tabular}

\section{Importancia de explicar y verbalizar}

En los relatos de situaciones cotidianas, encontramos que uno de los aspectos que los adultos más verbalizan y explican son las rutinas. Al tiempo que realizaban el baño, el aseo, la alimentación o las actividades previas para ir a dormir, también decían: vamos a bañarnos, qué rica sopa, es hora de ir a la cama, etc. Además de dichas verbalizaciones, las explicaciones emergían cuando no sólo se trataba de nombrar las cosas, sino de enseñar algo (cruzar la calle, lavarse los dientes).

\section{Por ejemplo, sobre dejar el pañal, los papás de Karina narraron:}

Entre los tres hicimos una despedida simbólica del pañal y le explicamos que ya era una niña más grande, que ya no iba a usar pañal, que éste lo iban a usar nenes más pequeños, como nena más grande ya ella iba a usar calzoncito y tener que usar su bacinica. Le encantó y la empezamos a llevar en la mañana: "aquí tienes que hacer pipí y popó". Al principio la llevábamos a cada rato y le insistíamos: "pipí, siéntate", ella lo captó muy rápido (Noé).

Cuando ella decía "pipí, popó" vamos inmediatamente y ya no deja que le bajemos la ropa interior, ella dice "yo, yo". Yo empecé a decirle que cómo se limpiaban las niñas e hicimos una especie de canción: que las niñas se limpian de adelante para atrás. Entonces lo repetíamos cada vez que iba al baño y ahora lo único que hago es preguntarle: "Nena, ¿cómo se limpian las niñas?" Y ella me dice "De adelante hacia atrás" (Gerardo).

Asimismo, notamos un significado compartido sobre la importancia de establecer horarios para que los niños y las niñas adoptaran una rutina, pues las y los participantes consideraron que esto les ayudaba a anticiparlas. Por ejemplo, Gabriela comentó que sus gemelos (los niños más pequeños de la muestra y por tanto, con lenguaje oral menos desarrollado) "entienden que es la hora de dormir, entienden que es su lugar". Resulta interesante el reconocimiento de las capacidades cognitivas, así como de sus alcances: "le decimos cómo debe lavarse sus genitales, sabemos que no lo entiende al $\mathbf{1 0 0} \%$, que no es algo que si se lo explicamos ya se pueda quedar [recordar], pero lo estamos haciendo de forma repetitiva" (Gerardo). Si bien, él consideró que su hija aún podría no comprender todo lo que se explicaba, eso no se significó como un impedimento para la enseñanza de hábitos.

También consideraron necesario enseñar desde temprana edad, reglas de cortesía (decir "por favor", "gracias", no hablar si se está comiendo, etc.). Gabriela comentó: "es importante inculcarles eso porque hay muchos niños que comen parados y corriendo, pero nosotras siempre los sentamos en la mesa". No obstante, al encontrar resistencia, los adultos refirieron el uso de la negociación, ofreciendo opciones: "ok, ¿estás llorando porque no te quieres ir a la cama todavía? 5 minutos más de juego y nos vamos a la cama" (Noé). Esto no significa que no haya cosas no negociables, pues consideran que seguridad y alimentación son asuntos esenciales: "no hay negociación si ella puede correr riesgo" (Flor).

En la última expresión de Noé o en la siguiente, puede verse también que emociones y sentimientos se pueden objetivar a través del lenguaje:

"le decimos que la amamos, que la queremos". Los padres (probablemente también porque su hija era la más grande de la muestra), parecen ser conscientes de la importancia de nombrar también las propias emociones. Sobre esto, Gerardo comentó que su hija "tiene que saber que de pronto yo necesito también un espacio porque estoy molesto y así se lo digo".

Finalmente, un elemento que todos consideraron importante explicar, es que existen diferentes tipos de familias. Eugenia refirió haberlo hecho de la siguiente manera: 
"Tú mamá y yo nos queremos mucho. Hay diversidad en el mundo, hay familias que tienen dos mamás, o dos papás, o una mamá y un papá, hay familias con abuelitas o tíos, o solo papá o mamá. En esta familia hay dos mamás que los aman muchísimo y no hay ninguna diferencia en eso".

Parece ser que su intención es que los niños y las niñas internalicen que su tipo de familia es como cualquier otra, sabiendo que deben explicitar su configuración: "Karina, cuando empiece a ser consciente, tiene que saber que esto no es raro y no está sola" (Gerardo). Encontramos un nodo de tensión e incertidumbre a futuro, pues efectivamente les preocupa que sus hijos/as puedan sufrir algún tipo de rechazo, de entrada, en su contexto escolar:

\begin{abstract}
"No vamos a permitir la discriminación o el bullying hacia Karina" (Noé). En el caso de los padres, el temor se acompaña al hecho de que, a diferencia del resto de niñas/os que viven con su madre biológica, Karina fue entregada en adopción; lo que ya de por sí está rodeado de estigmas y estereotipos en nuestra cultura. Quizá por ello Noé y Gerardo piensan que Karina "conocerá su historia cuando su desarrollo lo permita".
\end{abstract}

En síntesis, las explicaciones/verbalizaciones referidas por los adultos, parecen haber tenido propósitos diferenciados: nombrar (objetivar su mundo físico, psicológico y social), anticipar rutinas y/o enseñar algo con fines de aprendizaje. También reconocieron la necesidad de ajustar su vocabulario y la forma de dirigirse, tomando en cuenta la edad de sus hijas/os; así como la importancia del diálogo, sobre todo en la negociación que se da al establecer reglas, límites y consecuencias.

\section{Reglas, límites y consecuencias}

A pesar de que las parejas consideraron que ha sido complicado establecer reglas, llegaron a acuerdos sobre cómo establecer límites, así como en el tipo de consecuencias en caso de no cumplirse. Gabriela y Eugenia coincidieron en que para establecerlos, decir "no" era esencial, sin dejar de lado la explicación de por qué es importante seguir ciertas normas, así como aplaudir las conductas deseadas. Al respecto, Noé comentó que la imitación es una buena herramienta: "Karina se lava los dientes porque ve cómo nos los lavamos nosotros, entonces así aprendió”. Además de la negociación, él consideraba que "ser asertivos es mejor que simplemente decir 'no' ya que en algún artículo leímos que no es bueno decirles no".

Todos manifestaron claridad y consenso entre pareja sobre cuáles son las reglas no negociables. Gerardo las llamó "Reglas de casa" y junto con Noé, refirió usar el tiempo fuera de forma contingente y acompañado de explicaciones como una estrategia para evitar conductas indeseadas:

La llevamos a un lugar de la casa donde se tiene que quedar unodos minutos y siempre le decimos que se quede ahí pensando en que lo que hizo; que estuvo mal. Le decimos: "tiraste el plato y tiraste la comida y la comida no se desperdicia”, entonces le explicamos por qué está ahí y que tiene que pensar en lo que estuvo mal y hasta que se calme, deje de llorar, o de hacer el berrinche, ya podemos hablar. Luego le volvemos a explicar: "mi amor te puse ahí por esto, porque está mal lo que hiciste, porque no debes hacer esto” (Noé).

En correspondencia, Gerardo expresó que al establecer límites era necesario "mostrarme un tanto... pues inexpresivo, ni para bien ni para mal, que no perciba de mí que me estoy riendo ni que tengo el mayor coraje de mi vida", pues pensaba que así Karina podía discernir las cosas que hace.

No pegar fue una regla importante para las madres de los gemelos y Eugenia también narró, aunque sin nombrarla así, la estrategia del tiempo fuera, considerando que en ocasiones (ante peleas, gritos, ir a lugares donde no podían pasar) era necesario castigarlos: "los llevamos a su cuarto a que se calmen, se relajen y lo entienden muy bien, entonces así es como ponemos reglas". Finalmente, encontramos un consenso entre Flor y Lilia al pensar que el condicionamiento podría ser más eficaz que el castigo, lo cual se percibió como "muy cansado" para Flor. Ella agregó: "si haces esto, vamos a este lugar; sí vemos la caricatura, pero te comes la sopa de verduras".

En síntesis, los adultos describieron formas distintas para establecer reglas, límites y explicar consecuencias, pero consideraron fundamental el acuerdo entre pareja para trabajar en conjunto, aunque resulte complicado y desgastante, principalmente con los berrinches. Además, consideraron importante la coherencia que se transmite a través de la verbalización, pues esperaban que sus hijas/os fueran capaces de comprender por qué sus madres/padres realizaban diversas medidas.

\section{Enseñanza y aprendizaje de valores y habilidades sociales}

Considerando las diversas situaciones que en estos años generan ansiedad (ausencia de figuras parentales, ir a la guardería o escuela), para los adultos fue importante que aquellos/as aprendieran a esperar y tolerar su ausencia. Por ejemplo, Lilia usaba la explicación para anticiparlea Julia lo que haría (ir a trabajar, a alguna clase), con quiénesse quedaría y cuánto tiempo. Noé agregaría que es fundamental aprender a tolerar la frustración, aunque junto con su pareja (probablemente por su tipo de profesión), fueron los únicos en explicarla en términos de "etapas de desarrollo":

Está aprendiendo a que se tiene que frustrar y ni modo así va a ser; no puede obtener lo que ella quiere y cuando quiere y va a haber cosas que no puede hacer por su edad. Eso implica "los terribles dos" según hemos investigado, en esta etapa todo les frustra, se enojan mucho, hacen berrinche y no entienden que por su edad tienen que pedir ayuda, todo lo quieren hacer solos.

El respeto, la responsabilidad, la tolerancia a la diversidad y el compartir fueron valores básicos para los adultos, quienes afirmaron que para fomentar su aprendizaje, las/los niñas/os debían vivirlo y verlo en sus padres y madres. Dar de comer a los peces como valor de responsabilidad, respetar el lugar en el que comen o reconocer las diferencias que existen en sus familias como parte del valor de la tolerancia, son algunos ejemplos. Por otro lado, se esperaba que sus hijos/ as aprendieran a compartir cosas distintas, por ejemplo: juguetes, comida, etcétera.

Asimismo, y con relación a lo que se comentaba sobre la importancia de explicar su tipo de familia, los padres y las madres manifestaron elegir cuidadosamente la escuela a la que asistirían sus hijos/as, argumentando que las instituciones educativas debían propiciar un ambiente de inclusión y respeto a la diversidad. Así lo narró Noé: "en la escuela había niños de todos los colores y países. Tenía compañeros peruanos, chinos y eso nos gustó mucho porque ese es el ambiente de diversidad que estamos buscando para ella, o sea, que vea que no hay diferencias". Eugenia lo relató así: 


\section{Significados de madres y padres mexicanos del mismo género en torno a la crianza}

Ha sido realmente fácil, somos ahorita, por lo menos abiertamente, la primera pareja en la escuela. Las autoridades muy abiertas, las maestras también. Ha sido una experiencia sumamente grata y que también va a ser digno de compartir con nuestra comunidad de familias homoparentales.

La independencia y la autosuficiencia se tradujeron en pequeñas acciones (dejar que se vistieran sin ayuda, llevar su plato a la cocina) para impulsar la confianza en los niños y las niñas ante su intención de hacer algo; como afirmó Gerardo, "me gusta que lo intente". Lo anterior se asoció a diversas situaciones descritas sobre la importancia de que sus hijos/as eligieran y tomaran pequeñas decisiones: el vaso en qué tomar, la ropa qué vestir, los libros qué contar. Estas habilidades se relacionaron directamente con los valores de respeto e inclusión que padres y madres intentaban promover, percibiéndolos como vías para la futura autonomía.

\section{Mantener una relación cercana}

Los relatos estaban llenos de descripciones sobre contacto y muestras de cariño de forma cotidiana: "yo la saludo...jugamos, nos hacemos cosquillas, nos damos besos" (Gerardo); "necesito darles un abrazo y así sea el abrazo número 95 del día, no me importa, se los doy...se acuestan en mi regazo, y nos abrazamos, acariciamos" (Gabriela); "somos como muy apapachosas" (Flor); "trato, cuando le digo algo cariñoso, [de] verla a los ojos, de reírme mucho con ella” (Lilia). El poner a su hija al centro, queda muy bien ilustrado en el siguiente fragmento de Lilia:

Creo que ella siente más la cercanía cuando te involucras en su juego. Si estoy jugando con ella, no dejo que nada me distraiga, no estar en el teléfono, no hacer otra cosa, lo cual también a veces como adulto cuesta mucho trabajo (Lilia).

Las/os participantes consideraron que involucrándose no únicamente en el juego, sino en las rutinas cotidianas de aseo, alimentación, vestido; llevarlos o recogerlos de la escuela, lograrían que sus hijas e hijos se sintieran queridas/os y parte fundamental de su familia. Eugenia expresó: "si me quieren enseñar algo nuevo que aprendieron, si quieren leer un cuento nuevo, si quieren leer el mismo cuento 500 veces, eso es lo que trato de hacer para estar cerca de ellos".

Empero, debido al poco tiempo que algunas parejas solían tener por sus demandas laborales, todas valoraron el papel de diversas figuras que constituyeron una red de apoyo importante para la crianza: desde la trabajadora doméstica que se encargaba de la limpieza o la preparación de alimentos, hasta miembros de la familia extendida (abuelas, tíos, primos) que también asumían las rutinas, el juego, etc. Gerardo lo sintetizó así: "mi hija es amada y querida por todas las personas que la rodean, porque aparte ésa es mi labor, el círculo de Karina debe de ser un círculo de amor y protección y ésa es mi responsabilidad como papá”.

La importancia de una relación cercana, también se vinculó con una idea de dar gusto a los/as niños/as. Respecto a la alimentación, los adultos consideraron importante que fuera saludable. Flor y Gabriela no sólo refirieron vigilar que los ingredientes sean los adecuados, sino también buscar que les gustara: "que siempre tengan la lechita caliente, rica, que esté la comida bien preparada, hacerles lo que les gusta comer" (Gabriela). La intención de complacerles en otros aspectos como ver su película favorita antes de dormir se acompañó de una flexibilización en las reglas; por ejemplo, no comer dulces hasta terminar de comer: "si quiere una galleta de chocolate, pero no ha comido, pues tiene su galleta y come a la vez, y tiene la galleta ahí con ella y de repente le da mordiditas, pero va comiendo" (Flor). El trato afectuoso no parecía impedir establecer reglas y negociar: "QQuieres el celular o quieres el iPad?, podemos negociar, tal vez, 5 minutos dependiendo la hora" (Noé).

Finalmente, y con relación a la independencia, la felicitación se consideró como una forma de expresar la felicidad o el orgullo que madres y padres sentían hacia sus retoños, ante el despliegue de cierto tipo de comportamientos y capacidades valoradas en su desarrollo: "felicito mucho la autonomía, cuando ella decide lo que necesita realmente" (Flor); "cuando comparten, cuando aprenden algo que hasta ellos mismos se sorprenden, cuando te ponen mucha atención, es un festejo, de ¡bravo!, les aplaudimos, los abrazamos, bailamos, brincamos" (Eugenia). Noé comentó:

Cualquier logro, por pequeño que sea, como "ya me lavé los dientes" o "ya fui a dejar mi ropa sucia al bote de la ropa" se le aplaude, se le agradece y se le dice que está bien hecho, todo, todo lo bueno que hace por pequeño o grande que sea, se le felicita.

Ubicamos la finalidad del reconocimiento y la valoración como una forma de motivar y fomentar la adquisición y mantenimiento de diversas conductas, pero también en un sentido de acercamiento y fomento de la auto-confianza.

\section{Rasgos/atributos personales valorados}

Con relación a lo analizado en la categoría previa, nos llama la atención cómo para padres y madres, la afectuosidad (ser cariñoso/a) y la alegría (ser juguetón/a) son rasgos que consideraron característicos en sus hijos e hijas. La mayoría se lo explicó como algo que se aprende a partir de recibir continuas muestras de contacto físico y señales de cariño: "Karina se acerca a ti y te da un abrazo apretado, y sientes muy lindo porque creo que así ve que es el trato entre Gerardo y yo" (Noé). No obstante, al menos Lilia parecía considerar una explicación más innatista: "Julia ya vino así del empaque, es una niña muy amorosa, muy cariñosa, que te abraza o besa sin que se lo pidas, que te dice que te quiere o que te ama sin que se lo pidas".

La alegría como rasgo atribuido se vinculó con ser físicamente activo/a. Mostrar energía al saltar, correr y en general realizar las actividades propias de su edad se consideró como sinónimo de bienestar y salud. Por ejemplo, Eugenia manifestó que en un período llegó a preocuparse porque Gabriel "no quería interactuar, casi no te ponía atención o si le ponías alguna actividad, no la hacía”; sin embargo, posteriormente comprendió que aunque sus hijos fueran gemelos, no tenían por qué comportarse igual ("Paulo era mucho más dinámico").

Finalmente, un elemento de significación gira en torno a la construcción de la feminidad y la masculinidad como atributos que madres y padres consideraron que sus hijos/as internalizarían poco a poco: "es algo que ellos solitos construyen" (Lilia). Eugenia y Gabriela (las madres de los varones) coincidieron en que: "no les imponemos ni nuestra feminidad, ni la masculinidad externa”. De este modo, para los adultos propiciar la convivencia con "figuras femeninas y masculinas" es necesario, pues de algún modo no pueden quedarse fuera de los mandatos socioculturales. Así, encontramos en los relatos que las niñas se pintaban las uñas, se vestían como princesas, escogían muñecas con vestidos rosas y que los niños jugaban con carritos.

Pese a esto, notamos esfuerzos deliberados por propiciar formas diferentes de juego y exploración de roles, asumiendo que es impor- 
tante romper con los estereotipos: "el $\mathbf{1 0 \%}$ los hemos comprado nosotras y todos los demás se los han regalado y pues obviamente son juguetes para niños, ha habido también unas muñequitas que se han encontrado, nosotras no tenemos ningún problema con que las agarren" (Gabriela). De hecho, es interesante cómo estas madres (las únicas con hijos varones), señalan su evitación hacia juegos violentos y "propios de los niños", pero respetando ante todo las preferencias de los gemelos. Eugenia menciona:

No pretendemos imponerles que unos juguetes tienen que ser para ellos y otros para niñas, o ciertos programas de televisión, libros o películas. Entonces en realidad tratamos de tener un enfoque integral con ellos, también si quieren jugar con una muñeca lo pueden hacer.

Así pues, los adultos consideraron que los comportamientos "femeninos" o "masculinos" se explicaban como un aprendizaje por imitación: "cuando llega su abuela o con las asistentes que tiene Gerardo en el Hospital, le encanta pintarse las uñas o pintarse la boca" (Noé, refiriéndose a su hija Karina). O como Lilia mencionó sobre Julia:

De repente le encanta jugar con las herramientas y los clavos, le encanta clavar con los martillos (ríe), yo pienso que es porque yo lo hago mucho, entonces de repente cuando me ve, igual le entra. También le encanta jugar con las muñecas, los colores rositas, colgarse collares, pulseras, pintarse, aunque ni Flor ni yo somos así de todos los días pintarnos.

En síntesis, interpretamos que para los padres y las madres lo central está en respetar los intereses de sus hijos e hijas, fomentando que realicen elecciones y priorizando su bienestar emocional, sin que ello implique "extraerlos" de su cultura y de su entorno social.

\section{Discusión y conclusiones}

Al ver las categorías en conjunto podemos identificar que la mayoría alude al papel del adulto, lo cual puede indicar que las y los entrevistados tienen una representación amplia de lo que la crianza implica, en términos de su función como madres y padres; es decir, qué acciones y propósitos son importantes en su desempeño como educador/a para propiciar el desarrollo integral de sus hijos/as.

Los relatos sobre todo de la primera categoría, dan cuenta de la intención adulta por reconocer el momento de desarrollo de sus hijos e hijas, ajustando su lenguaje en función de lo que podrían ser capaces de comprender. Explicitar las emociones tanto de sus hijos/as como las propias, es relevante en tanto que el desarrollo de una teoría de la mente se ha relacionado con la empatía (Zegarra-Valdivia \& Chino, 2017). Es como si supieran lo importante que es "prestar su mente" en forma de lenguaje, para explicitar y objetivar el mundo. Estas habilidades parentales pueden relacionarse con su alto nivel socio-educativo, pues como ha encontrado Triana (2003):

Los padres de niveles profesionales medios y altos, así como los que residen en zonas urbanas...se sienten más protagonistas y responsables de la educación de sus hijos, considerando que su actuación tiene una enorme repercusión sobre ellos. Además...ven a los niños como seres competentes y capaces que pueden actuar modificando los efectos ambientales (p.230).
Aunado al alto nivel socio-educativo de nuestros participantes, un elemento común es la red de apoyo con que cuentan para la crianza, constituida por abuelos, tíos y personal de apoyo; hallazgo que coincide con lo reportado por González y López (2009). La red de apoyo como contexto interactivo parece ser un ingrediente fundamental tanto en la crianza como en los significados que se construyen sobre esta y el desarrollo. Como señala Bruner (1998), las concepciones evolutivas, una vez apropiadas por la cultura se constituyen en una realidad social que configura los propios fenómenos evolutivos.

Llama la atención el delicado equilibrio entre la intención de complacerles y darles gusto, incluyendo la satisfacción de sus necesidades básicas (alimentación, sueño, etcétera) y el fuerte trabajo que subyace a enseñar reglas, límites y valores. La primera idea (importancia de complacerlos) no parece vincularse con permisividad, pues hay que recordar que este estilo se caracteriza por ausencia de límites (Jorge \& González, 2017). Más bien parece relacionarse con la importancia del vínculo afectivo, así como con los rasgos valorados en sus niñas/ os (alegría, calidez). Este significado de crianza afectuosa puede relacionarse con la idea de seguridad y protección hacia los/as niños/as; de ahí que para estos padres y madres fuera evidente la necesidad de establecer límites y reglas claras.

Éstas no se observan arbitrarias y de hecho representan una variedad de estrategias sin tener que recurrir al castigo físico o verbal, el cual sigue presente en nuestra cultura (Morales, Félix, Rosas, López, \& Nieto, 2015; Sauceda-García, Olivo-Gutiérrez, Gutiérrez, \& MaldonadoDuran, 2007; Vite \& López, 2010). Por el contrario, nosotros encontramos que si bien era importante establecer consecuencias, las formas de modificación conductual descritas fueron otras: tiempo fuera, explicación, negociación, reforzamiento positivo y modelado.

De acuerdo con Pulido et al. (2013), existe una cuestión generacional que permitiría explicar por qué los padres/madres jóvenes en general, recurren a otras formas de instrucción diferentes al castigo. Sin embargo, nuestras/os participantes estaban en plena madurez y más bien lo que parecía imperar es una visión más amplia de las niñas y los niños como personas con derechos, a quienes se les fomenta la autonomía y con quienes se puede dialogar y negociar pese a su corta edad.

Consideramos que una parte importante de los límites y reglas tenían que ver con normas de convivencia, lo cual puede relacionarse con ambientes libres de violencia. Encontramos un sentido de acciones recíprocas entre adultos y pequeños; es decir, los padres gais y las madres lesbianas parecían tener muy clara la necesidad e importancia de enseñar valores y, en ese sentido, intentaban poner el ejemplo y felicitar a sus hijos e hijas cuando manifestaban aprenderlos.

Lo anterior no significa que la tarea cotidiana de establecer normas y límites no sea un enorme trabajo exento de dificultades, pues como nuestras/os participantes reconocieron, es una de las dimensiones más desgastantes de la crianza. Como afirman Infante y Martínez (2016), tener consciencia de que las reglas son importantes para mantener el orden, el respeto y la armonía familiar, no implica que sea una tarea sencilla.

Además, los relatos sobre este tema, reflejaron un importante consenso entre la pareja, todo lo cual, coincide con lo reportado por González et al. (2013), sobre una crianza más democrática entre parejas homosexuales. Los hijos y las hijas fueron concebidos como personas con voz y decisión que tienen la capacidad de elección y de ser responsables. Algunos de los participantes parecían identificar de forma adecuada el nivel de complejidad que requieren las tareas, deberes o peticiones a sus hijas e hijos en función de su momento de desarrollo. 


\section{Significados de madres y padres mexicanos del mismo género en torno a la crianza}

De ahí que también fue importante para aquéllos que pese a su corta edad, sus pequeños/as pudieran aprender a tolerar la frustración y a ser poco a poco más autónomos/as.

La apuesta por la autonomía e independencia en la crianza, nos parece muy relevante pues en nuestra cultura, al igual que en muchas otras de Latinoamérica, se tiende a sobreproteger a las/os pequeñas/ os. Como encontraron Goncalvez y Franco (2011), adultos heterosexuales ejercían un estilo permisivo, que al menos en el contexto de esa provincia venezolana, se asoció con una cosmovisión del niño como alguien indefenso o débil. Consideramos que en última instancia son las concepciones culturales (y no la orientación sexo-afectiva de padres y madres) lo que configura el mundo simbólico que se teje alrededor de lo que significa ser pequeño/a.

Más que débiles o indefensos, nuestros participantes situaron a sus hijos e hijas como personas activas y dinámicas, lo cual puede relacionarse con asistir a la escuela. Por ejemplo, Goncalvez y Franco (2011) también encontraron que a partir de la entrada al preescolar, los niños eran percibidos por las personas adultas como más fuertes y seguros. Y sin duda, la educación inicial tiene un impacto en el desarrollo y en concreto, en la adquisición/consolidación de hábitos, rutinas y disciplina que contribuyen en la autonomía.

En nuestros participantes, este interés por formar personas independientes parece tener que ver también con el miedo a futuro de que sus hijos e hijas pudieran sufrir algún tipo de discriminación por la forma en que está constituida su familia. Este hallazgo es similar a lo reportado por Laguna (2016), con quien también coincidimos en el cuidado que manifestaron nuestros padres y madres al elegir escuela, en el sentido de buscar un entorno protector y con una ideología de respeto a la diversidad.

El hecho de ser hombres gais y mujeres lesbianas luchando para que su tipo de familia sea reconocido, no les eximió de elaborar la feminidad y masculinidad de sus críos en términos de ciertos estereotipos de género. Después de todo, cualquier familia refleja de algún modo el sistema de valores imperante ya que no puede vivirse "fuera" de la cultura. Con todo y muy probablemente relacionado a su nivel educativo y orientación sexo-afectiva, nuestros participantes dan indicios de altos niveles de reflexividad sobre su labor como padres y madres, lo cual podría permitirles tomar consciencia sobre sus creencias y acciones, en miras de mejorarlas.

Según Holden, Kozak, Smith, Singh y Ashraf, (2017), la crianza efectiva implica múltiples procesos cognitivos sociales, incluido el conocimiento del/a niño/a individual, la crianza propia, la resolución adecuada de problemas, la flexibilidad en las respuestas y las evaluaciones frecuentes del/a niño/a. Los padres con mayores habilidades metacognitivas, o dicho de otro modo, que son más reflexivos y conscientes sobre sus acciones, suelen ejercer una disciplina mucho más razonada y deliberada, en lugar de una reactiva y coercitiva. Dado nuestro objeto de estudio, no tenemos elementos para afirmar algo sobre su crianza como tal, pero sí para identificar un nivel de coherencia entre lo que reflexionan de forma más amplia y lo que narran de sus experiencias cotidianas.

Nuestros hallazgos coinciden con lo encontrado por Contreras y Pons (2015), sobre los roles de crianza flexibles y negociadores en cinco familias homoparentales. De forma similar, en nuestras tres parejas observamos un esfuerzo intencional por tomar consciencia sobre las prácticas de crianza que están utilizando, así como una reflexión y genuina preocupación sobre el impacto de las mismas en el comportamiento de sus hijos/as.
En conclusión, los significados sobre la crianza de nuestros padres gais y madres lesbianas reconstruidos a partir de su narración de experiencias cotidianas, hacen justicia al significado socialmente más compartido de la crianza, en términos de cuidado, protección y educación. Ellas y ellos saben de la importancia de una buena alimentación, del fomento de hábitos, de establecer rutinas, normas, reglas y consecuencias; de las ventajas de la negociación, explicación, modelar con el ejemplo y enseñar valores como la autonomía y el respeto, considerando a las niñas y los niños como individuos con derechos. Además, conocen sobre lo fundamental que son las demostraciones de afecto para propiciar un entorno cálido y amoroso a sus hijos e hijas, reconociendo la participación de otras figuras de apoyo que constituyen los contextos interactivos más próximos.

Es necesaria mayor investigación con padres y madres homosexuales, que residan no sólo en la Ciudad de México, o zonas urbanas, sino en contextos más desfavorecidos, tanto económicamente, como en términos de capital cultural. Es preciso conocer cómo se significa y experimenta la crianza en familias que no cuentan con una red de apoyo, con un bajo nivel educativo o donde los grupos sociales más próximos muestran prejuicios y estereotipos hacia las familias homoparentales.

\section{Referencias}

Ainsworth, M., Blehar, M. C., Waters, E., \& Wall, S. (1978). Patterns of attachment. Hillsdale, new york : NJ: Erlbaum. Retrieved from http:// parentalalienationresearch.com/PDF/2015ainsworth.pdf

Arcila, P. A., Mendoza, Y. L., Cañón, O. E., \& Jaramillo, J. M. (2010). Comprensión del significado desde Vygotsky, Bruner y Gergen. Diversitas: Perspectivas en Psicología, 6(1), 37-49. doi:10.15332/s17949998.2010.0001.03.

Berger, R. (2013). Now I see it, now I don't: researcher's position and reflexivity in qualitative research. . Qualitative research, 1-16. doi:doi: 10.1177/1468794112468475

Bowlby, J. (1996). Una base segura: aplicaciones clínicas de una teoría del apego. barcelona: paidos. Retrieved from https://dialnet.unirioja.es/ servlet/libro? codigo $=50244$

Braun, V., \& Clarke, V. (2006). Using thematic analysis in psychology. Qualitative research in psychology, 3(2), 77-101. doi:doi: http://dx.doi. org/10.1191/1478088706qp0630a

Bruner, J. (1990). Actos de significado. Más allá de la revolución cognitiva. madird: alianza. Retrieved from https://dialnet.unirioja.es/servlet/ libro?codigo $=278788$

Bruner, J. (1998). La teoría del desarrollo como una cultura. In Realidad mental y mundos posibles. Los actos de la imaginación que dan sentido a la experiencia (pp. 138-152). Barcelona: Gedisa.

Carneiro, F., Tasker, F., Salinas-Quiroz, F., Leal, I., \& Costa, P. A. (2017). Are the fathers alright? A systematic and critical review of studies on gay and bisexual fatherhood. Frontiers in Psychology. , 8, 1636. doi:doi: 10.3389/fpsyg.2017.01636

Contreras, J. B., \& Pons, L. (2015). Familias en transformación: experiencias de parejas diversas que promueven la ruptura de roles tradicionales en la crianza de sus hijos e hijas. In J. Medina, Familias Homoparentales en México: mitos, realidades y vida cotidiana (pp. 85-110). MEXICO: Letra S, Sida, Cultura y Vida Cotidiana, A.C. Retrieved from http://189.240.117.226/biblos-imdf/node/14866

Cuervo, A. (2010). Pautas de crianza y desarrollo socioafectivo en la infancia. Diversitas. Perspectivas en psicología, 6(1), 111-121. doi:doi: 10.15332/s1794-9998.2010.0001.08

Da Rocha, E., Roethle, M., \& Mochizuki, A. (2012). Metas de socialización en diferentes contextos. Paidéia, 33-42. doi:http://dx.doi.org/10.1590/ S0103-863X2012000100005. 
Denzin, N. K., \& Lincoln, Y. S. (2012). Manual de Investigación Cualitativa. . Barcelona: Gedisa. Retrieved from https://dialnet.unirioja.es/servlet/ libro? codigo $=490631$

Duarte, L., García, N., Rodríguez, E., \& Bermúdez-Jaimes, M. E. (2016). Las prácticas de crianza y su relación con el vínculo afectivo. Revista Iberoamericana de Psicología, 9(2), 113-124. Retrieved from https:// reviberopsicologia.ibero.edu.co/article/view/

Fedewa, A., Black, W., \& Ahn, S. (2015). Children and adolescents with same-gender parents: A meta-analytic approach in assessing outcomes. Journal of GLBT Family Studies, 11, 1-34. doi:doi:1080/1550428X.2013.869486

Flick, U. (2004). Introducción a la investigación cualitativa. madrid: morata. Retrieved from https://www.buscalibre.com.co/libro-introducciona-la-investigacion-cualitativa-uwe-flick-morata/9788471124807/p/33 30409? gclid=EAlalQobChMlj9arz5W56QIV5QilCR3USgYREAYYASABEg KWY D BWE

Giraldo, A. S. (2015). Azares en las prácticas de paternidad de algunos hombres gay de México, D.F. y en el acercamiento a su estudio. En: J. A. Medina (Comp.) Familias Homoparentales en México: mitos, realidades y vida cotidiana. In A. Rosales, \& E. Tapia, Sexualidades y géneros imaginados:educación, políticas e identidades lgbt (pp. 6384). MEXICO ,D.F: UNIVERSIDAD PEDAGOCIA NACIONAL . R

Goncalvez, D., \& Franco, F. (2011). Las pautas de crianza en la ciudad de Mérida y su relación con la educación inicial. Registro etnográficoexploratorio. Revista de Teoría y Didáctica de las Ciencias Sociales, 1(17), 165-192. Retrieved from https://www.redalyc.org/articulo. oa?id $=65221619011$

González, M. M., \& López, F. (2009). Relaciones familiares y vida cotidiana de niños y niñas que viven con madres lesbianas o padres gays. . Cultura y educación, 21(4), 417-428. doi:10.1174/113564009790002364

González, M. M., Diez, M., López, F., Martínez, E., \& Morgado, B. (2013). Diversidad familiar y estrategias de conciliación en Andalucía. Proyecto Diversia.Instituto Andaluz de la MujeR. SEVILLA: Trama Gestión, S.L. Retrieved from http://www.juntadeandalucia.es/iam/ catalogo/doc/iam/2013/143363433.pdf

Haces, M. A. (2015). La Homoparentalidad en perspectiva. In J. A. Medina, Familias Homoparentales en México: mitos, realidades y vida cotidiana (pp. 27-38). México, D.F: Letra S, Sida, Cultura y Vida Cotidiana.

Harkness, S., Zylicz, P. O., Super, C. M., Welles-Nyström, B., Bermúdez, M. R., Bonichini, S., \& Mavridis, C. J. (2011). Children's Activities and Their Meanings for Parents: A Mixed-Methods Study in Six Western Cultures. Journal of Family Psychology, 25(6), 799-813. doi: doi:10.1037/a0026204

Holden, G. W., Kozak, C., Smith, M. M., Singh, J. P., \& Ashraf, R. (2017). Disciplinary practices, metaparenting, and the quality of parent-child relationships in African-American, Mexican-American, and EuropeanAmerican mothers. International Journal of Behavioral Development, 41(4), 482-490. doi:10.1177/0165025416687414

Infante, A., \& Martínez, J. F. (2016). Concepciones sobre la crianza: el pensamiento de madres y padres de familia. LIBERABIT. Revista Peruana De Psicología, 22(1), 31-41. doi:doi:10.24265/liberabit.2016. v22n1.03

Isaza, L., \& Henao, G. C. (2010). El desempeño en habilidades sociales en niños de dos y tres años de edad, y su relación con los estilos de interacción parental. Electronic Journal of Research in Educational Psychology, 8(3), 1051-1076. doi:doi:10.25115/ejrep.v8i22.1453

Jorge, E., \& González, M. C. (2017). Estilos de crianza parental: una revisión teórica. Informes Psicológicos, 17(2), 39-66. doi:doi:10.18566/infpsic. v17n2a02

Laguna, O. E. (2015). Parentalidad gay en la Ciudad de México: de la negación impuesta a la desestabilización involuntaria. En: J. A. Medina. In J. Medina, Familias Homoparentales en México: mitos, realidades y vida cotidiana (pp. 39-62). MEXICO: Letra S, Sida, Cultura y Vida Cotidiana, A.C. doi:http://189.240.117.226/biblos-imdf/ node/14866
Laguna, O. E. (2016). Arreglos parentales de varones gay en la Ciudad de México: de la paternidad negada a la transformación inadvertida del cuidado. Masculinities and Social Change, 5(2), 182-204. doi:10.17583/ MCS.2016.2033

Maxwell, J. A. (1996). Qualitative research design. An interactive approach. California:: Sage Publications, Applied Social Research Methods Serie. Retrieved from https://www.amazon.com/-les/Joseph-Maxwell/. dp/1412981190

Merrifield, K. A., Gamble, W. C., \& Jin, J. (2015). Using social cognitive theory to understand meta-parenting in parents of young children. Family Science, 6(1), 362-369. doi:doi:10.1080/19424620.2015.1076495

Mone, I., Benga, O., \& Susa, G. (2014). The relationship between cultural model, socialization goals and parental ethnotheories: A mixed method study. Cognition, brain and behavior, 23(3), 191-208. Retrieved from https://www. researchgate.net/publication/270215480 The relationship between cultural model socialization goals and parental_ethnotheories_A_mixed_method_study

Morales, S., Félix, V., Rosas, M., López, F., \& Nieto, J. (2015). Prácticas de crianza asociadas al comportamiento negativista desafiante y de agresión infantil. Avances en Psicología Latinoamericana, 33(1), 57-76. doi:10.12804/ap/33.01.2015.05

Morgante, M. G., \& Remorini, C. (2013). Estudio etnográfico de la crianza y de la participación de los niños en comunidades rurales de los Valles Calchaquíes septentrionales (noroeste Argentino). Bulletin de l'Institut français d'études andines, 42(3), 411-433. doi:https://doi. org/10.4000/bifea.4177

Otzen, T., \& Manterola, C. (2017). Técnicas de muestreo sobre una población a estudio. Journal Morph, 35(1), 227-232. doi:doi: 10.4067/S0717$\underline{95022017000100037}$

Pulido, S., Castro-Osorio, J., Peña, M., \& Ariza-Ramírez, D. P. (2013). Pautas, creencias y prácticas de crianza relacionadas con el castigo y su transmisión generacional. Revista Latinoamericana de Ciencias Sociales, Niñez y Juventud, 11(1), 245-259. doi:doi:10.11600/1692715x.11116030912

RealAcademiaEspañola. (2018). Diccionario de la lengua española (edición del tricentenario). Consultado el 12 de julio de 2019. Retrieved from https://dle.rae.es/criar?m=form

Rodrigo, M. J. (2003). Representaciones y procesos en las teorías implícitas. En: M. J. Rodrigo, A. Rodríguez y J. Marrero. (Coords.). Las teorías implícitas. Una aproximación al conocimiento cotidiano. madrid: visor. Retrieved from https://www.researchgate.net/ publication/311533920 Las teorias implicitas Una aproximacion al conocimiento cotidiano

Rogoff, B. (1993). Aprendices del pensamiento: el desarrollo cognitivo en el contexto social. barcelona : paidos. Retrieved from https://www.todostuslibros.com/libros/aprendices-delpensamiento 978-84-7509-896-8

Roncancio-Moreno, M., Bermúdez-Jaimes, M. E., \& Uchoa, A. (2019). El desarrollo del sí mismo en la infancia: Contribuciones teóricas y metodológicas desde la teoría del self dialógico (TSD) y la psicología semiótico-cultural. Revista Iberoamericana de Psicología, 12(1), 7788. doi: 10.33881/2027-1786.rip.12107

Salinas-Quiroz, F., \& Costa, P. A. (2018). Arreglos parentales de personas lesbianas, gais, bisexuales y trans (LGBT): estado del arte. En: A. L. Rosales y E. Tapia (Coords.). Sexualidades y géneros imaginados: educación, políticas e identidades LGBT. In A. Rosales, \&E. Tapia, Sexualidades y generos imaginados:educacion politicas e identidades lgtb (pp. 215-246). Ciudad de mexico: horizontes educativos. Retrieved from https://www.researchgate.net/ publication/324845101_Arreglos_parentales_de_personas_lesbianas gais bisexuales y trans LGBT estado del arte

Salinas-Quiroz, F., Rodríguez, F., Costa, P. A., Rosales, M., Silva, P., \& Cambón, V. (2018). Can Children Have Ordinary Expectable Caregiving Environments in Unconventional Contexts? Quality of Care Organization in Three Mexican Same-Sex Planned Families. Frontiers in Psychology, 9, 2349. doi: doi:10.3389/fpsyg.2018.02349 


\section{Significados de madres y padres mexicanos del mismo género en torno a la crianza}

Sandín, M. P. (2003). El rigor científico en la investigación cualitativa. In Investigación cualitativa en educación. Fundamentos y tradiciones (pp. 185-201). Madrid: McGraw Hill.

Sauceda-García, J. M., Olivo-Gutiérrez, N. A., Gutiérrez, J., \& MaldonadoDuran, J. M. (2007). El castigo físico en la crianza de los hijos. Un estudio comparativo. Boletín médico del Hospital Infantil de México, 63(6), 382-388. Retrieved from http://www.scielo.org.mx/scielo. php?script $=$ sci arttext\&pid $=$ S1665-11462006000600004

Tarrés, M. L. (2001). Lo cualitativo como tradición. In M. Tarres, Observar, escuchar y comprender sobre la tradición cualitativa en la investigación social (pp. 35-60). MEXICO: FLACSO. Retrieved from https://www.amazon.com/Observar-comprender-tradici\%C3\%B3ncualitativa-investigaci\%C3\%B3n-ebook/dp/B00JODVFU2

Triana, P. B. (2003). Las teorías implícitas de los padres sobre la infancia y el desarrollo. En: M. J. Rodrigo, A. Rodríguez P. y J. Marrero. (Coords.) Las teorías implícitas. Una aproximación al conocimiento cotidiano . madird : visor. Retrieved from https://dialnet.unirioja.es/servlet/ tesis?codigo $=135301$
Vite, A., \& López, F. (2010). La asincronía materna autoritaria y permisiva en el maltrato infantil. Revista mexicana de análisis de la conducta, 36(3), 23-34. doi:10.5514/rmac.v36.i3.02

Vygotsky, L. S. (1934/1995). Pensamiento y lenguaje. Barcelona: Paidós. Retrieved from https://www.todostuslibros.com/libros/pensamiento-ylenguaje_978-84-493-3680-5

Weiss, H. E. (2007). Hermenéutica y descripción densa vs teoría fundamentada. Revista Mexicana de Investigación Educativa, 22(73), 637-654. Retrieved from https://www.redalyc.org/ pdf/140/14050493013.pdf

Zegarra-Valdivia, J., \& Chino, B. (2017). Mentalización y teoría de la mente. Revista de neuropsiquiatría, 80(3), 189-199. doi:10.20453/rnp. v80i3.3156 